\title{
Development of Reconfigurable Frequency Selective Surface Associating Cross Dipoles and Matryoshka Geometries
}

\author{
Alfredo Gomes Neto (D), Jefferson Costa e Silva (D), Ianes Barbosa Grécia Coutinho (D), \\ Diego Cássio Garcia Fernandes (), Laryssa Maria de Sousa Duarte (), Amanda Gomes Barboza (D) \\ Group of Telecommunications and Applied Electromagnetism, GTEMA \\ Federal Institute of Paraiba, IFPB, Brazil \\ alfredogomes@ifpb.edu.br,jefferson@ifpb.edu.br,ianesgrecia@gmail.com,diegocassio34@gmail.com, \\ maria.laryssa@gmail.com,amanda_bgomees@hotmail.com
}

\begin{abstract}
A reconfigurable frequency selective surface, FSS, associating cross dipoles and matryoshka geometries is described in this paper. The proposed reconfigurable FSS uses as active element PIN diodes. With the associated geometries, three resonant frequencies are achieved, two fixed, related to the matryoshka geometry, and one that can be activated or not, related to the cross dipoles geometry. Differently from previous works, an inductor was inserted into the cross dipoles' horizontal arms, maintaining its resonance for $X$ polarization. The PIN diode inserted into cross dipoles' vertical arms controls the respective resonance. The design procedures for each geometry, in addition to PIN diode and RF inductor basics are outlined. The expected results are discussed based on the analysis of the resonances of each geometry and the associated geometries, as well as the different PIN diode bias states (OFF and $\mathrm{ON}$ ). A prototype was fabricated and characterized, obtaining a good agreement between the numerical and experimental results, with a reconfiguration bandwidth of $0.24 G \mathrm{~Hz}$, from $2.12 \mathrm{GH} z$ to $2.36 \mathrm{GH}$, for a variation of at least $10 \mathrm{~d} B$ between the OFF and ON states.
\end{abstract}

Index Terms—Cross dipoles, matryoshka, PIN diode, reconfigurable FSS.

\section{INTRODUCTION}

With increasing number of users and diversity of services, telecommunications systems, especially mobile communication systems, use the frequency spectrum more and more intensively. Artificial intelligence (AI), cloud-based computing, Internet of Things (IoT), big data, cyber security and resilience, robotic process automation and fifth-generation mobile communications system $(5 \mathrm{G})$ are just some examples of the existing demands, requiring an adequate management of the radio frequency spectrum, imposing restrictions both in the frequency bands to be used, as in the power levels [1]. These restrictions have required new solutions, such as reconfigurable antennas [2]-[4] and electromagnetically intelligent buildings [5], [6], in order to optimize the use of the spectrum and minimize interference.

In the implementation of reconfigurable antennas, as well as electromagnetically intelligent buildings, one of the adopted approaches is the use of frequency selective surfaces, FSS, in its Brazilian Microwave and Optoelectronics Society-SBMO received 28 Jan 2021; for review 28 Jan 2021; accepted 20 Mar 2021 
reconfigurable form, reconfigurable frequency selective surfaces [2]-[7]. Essentially, a FSS consists of conductive patches, or aperture elements, etched on a dielectric substrate, arranged in a planar periodic structure, with the property of filtering electromagnetic waves as a function of frequency. As shown in Fig.1, the frequency response of the FSS depends on the characteristics of the substrate, the geometry and periodicity of the unit cells, and the polarization of the incident wave [7]-[9]. As FSS can let pass or block electromagnetic waves in free space for different frequencies, they are also known as spatial filters.

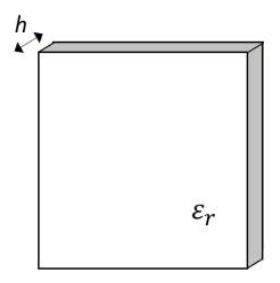

a)

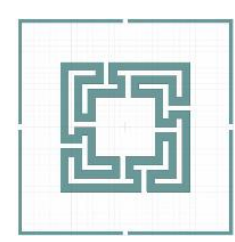

b)



c)



d)

Fig. 1. Parameters that affect the FSS frequency response: a) Substrate characteristics, b) Unit cell geometry,

c) Periodicity, d) Incident wave polarization.

In FSS reconfiguration two main approaches are used: mechanical reconfiguration and electronic reconfiguration. In mechanical reconfiguration, mechanical modifications are explored, such as stretching, bending or rotating the basic element to obtain the frequency response adjustment. On the other hand, in electronic reconfiguration, discrete components, such as varactors, PIN diodes or MEMS switches, are incorporated into the basic geometry of the FSS [2], [7]. In both cases, the variation of the frequency response can occur in discrete or continuous values, depending on the variation technique employed.

This paper describes the development of an electronically reconfigurable FSS, based on the association of cross dipoles and matryoshka geometries, with the PIN diode as active element. In this way, the obtained reconfigurable FSS operates in three frequency bands, two related to the matryoshka geometry and the third one to the cross dipoles. In this last one frequency band, the resonance is activated or not, accordingly PIN diode bias state (ON or OFF), modifying the FSS frequency response. To maintain the cross dipoles isolated and allow that polarization current pass to the PIN diodes, RF inductors are inserted into cross dipoles horizontal arms. In order to verify the proposed reconfigurable FSS behavior, a prototype was fabricated and characterized, confirming expected results.

After this Introduction, the reconfigurable FSS design is described in Section II, including geometries, PIN diode and RF inductor basics. The fabricated prototype and its characterization, with numerical and measured results for each design stage, are detailed and discussed in Section III. Results and conclusions are summarized in Section IV. 


\section{FSS DESIGN}

This section describes the cross dipoles and matryoshka geometries, as well as the model used for the PIN diode. Furthermore, basics of RF inductors are also introduced. The expected results are discussed, before and after the insertion of the PIN diodes and RF inductors. The substrate is considered to have a thickness $h$ and a dielectric constant $\varepsilon_{r}$.

\section{A. Cross dipoles geometry}

Cross dipoles geometry, shown in Fig. 2, is one of the simplest geometries, for which the resonance frequency occurs when the dipole length is approximately half guided wavelength [7], [8]. Its resonance frequency can be estimated from (1) [9], [10].



Fig. 2. Cross dipoles geometry.

$$
L_{d i p}=\frac{3 \times 10^{8}}{2 f_{\text {res }} \sqrt{\varepsilon_{\text {ref-dip }}}}
$$

in which $f_{r e s}$ is the desired resonance frequency and $\varepsilon_{r e f-d i p}$ is given by:

$$
\varepsilon_{\text {ref-dip }}=\frac{\varepsilon_{r e f-M S}+\varepsilon_{r e f-C P W}}{2} .
$$

where:

$\varepsilon_{r e f-M S}$ is the effective dielectric constant for a microstrip, considering the microstrip width equal the dipole width, $w$, and a dielectric thickness $h$.

$\varepsilon_{r e f-C P W}$ is the effective dielectric constant for a coplanar waveguide without ground plane, with $s=10 \times h$ and the center strip width equal to the dipole width, $w$ [11].

$\varepsilon_{r e f-M S}$ and $\varepsilon_{r e f-C P W}$ that can be easily calculated using one of the available software [12], [13].

\section{B. Matryoshka geometry}

The matryoshka geometry in Fig. 3 was introduced in [14], [15]. Differently from concentric rings, in matryoshka geometry the rings are interconnected, increasing its effective length and presenting, consequently, characteristics of miniaturization and multiband operation. However, this geometry was polarization dependent. In [16], [17] a polarization independent matryoshka geometry was proposed, as seen in Fig. 4, and it is the reference for the geometry considered in this paper. The square matryoshka ring is adopted, as it is easier to manufacture than circular one. 


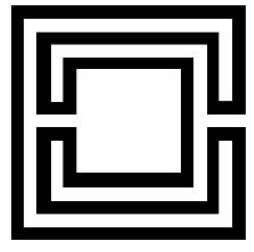

a)

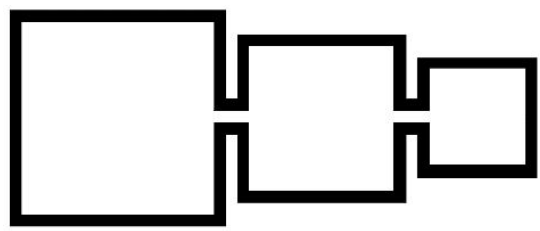

b)

Fig. 3. Matryoshka geometry: a) Matryoshka ring, b) Expanded matryoshka ring.



a)

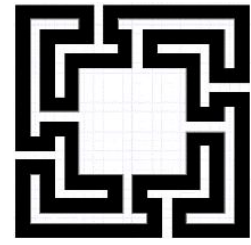

b)

Fig. 4. Polarization independent matryoshka geometry: a) Matryoshka circular ring, b) Matryoshka square ring

The matryoshka geometry is obtained by the following steps. Initially, concentric square rings are designed, as depicted in Fig. 5(a). Then, gaps are inserted at the same position in consecutive rings as Fig. 5(b). Finally, the consecutive rings are connected and the matryoshka ring is achieved in Fig. 5(c). Usually, $L x i=L y i=L i, d x i=d y i=d i, i=1,2,3$. The unit cell periodicity is determined by the filling factor, grating lobes limitations etc. However, in this paper the cross dipoles lengths determine the unit cell periodicity.



a)

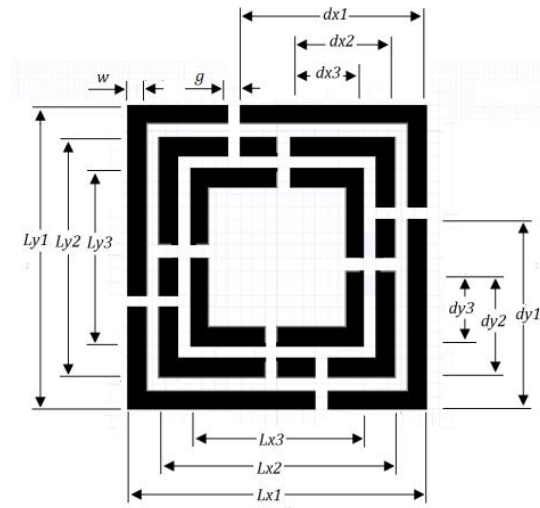

b)



c)

Fig. 5. Polarization independent matryoshka geometry step by step: a) Concentric square rings, b) Concentric square rings with gaps, c) Matryoshka square ring geometry.

The matryoshka geometry presents a multi-resonant behavior [14]-[17], but in this paper only the first two resonant frequencies are considered. Analogues to cross dipoles geometry, the equations (3) - (6) provide a first approach for the two first resonant frequencies.

1. Matryoshka geometry - first resonant frequency:

$$
f_{\text {res } 1}=\frac{3 \times 10^{8}}{L_{e f 1 \sqrt{\varepsilon_{r e f-C P W}}}}
$$

with

$$
L_{e f 1}=3\left(L_{1}-2 w\right)+2\left(L_{2}-2 w\right)+3\left(L_{3}-2 w\right)
$$


2. Matryoshka geometry - second resonant frequency:

$$
f_{\text {res } 2}=\frac{3 \times 10^{8}}{\left(L_{e f 2} / 2\right) \sqrt{\varepsilon_{\text {ref-CPW }}}},
$$

with

$$
L_{e f 2}=3 L_{1}+2 L_{2}+3 L_{3}
$$

It must be emphasized that (1) - (6) are initial design equations, a first step towards a numerical optimization. Furthermore, the incident wave is considered normal to the $\operatorname{FSS}\left(\theta=0^{\circ}\right)$.

\section{PIN diode}

The PIN diode is a semiconductor device that operates as a variable resistor in RF and microwave applications. In general, two operating points of the PIN diode are considered, the forward polarization (low impedance) and the reverse polarization (high impedance), as illustrated in Fig. 6 [18]. In spite of a more accurate PIN diode model includes inductances, capacitances and resistances, as shown in Fig. 7, in many applications a simplified model, considering only the variable resistance, can be adopted. In this work the PIN diode BAR 64-03W was used, and its principal characteristics are presented [19].

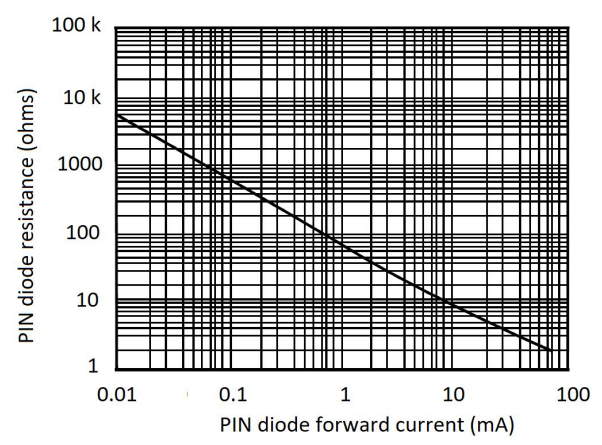

Fig. 6. Typical PIN diode resistance as a function of forward current.



a)

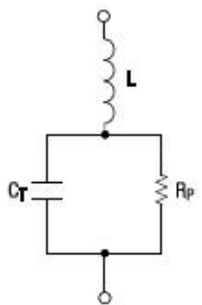

b)

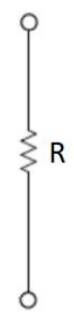

c)

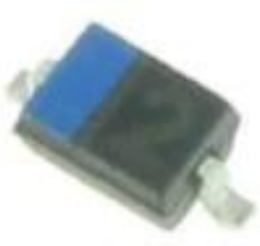

d)

Fig. 7. PIN diode models: a) Forward bias model, b) Zero or reverse bias model, c) Simplified model, d) PIN diode BAR 64$03 \mathrm{~W}$.

\section{RF inductor}

Inductors are key circuit components in many electronic designs, from power and voltageconversion circuits to higher-frequency RF and microwave circuits. Apart of its inductance, in RF and microwave applications parameters, such as DC resistance (DCR), quality factor (Q) and self-resonant frequency (SRF) are performance parameters that may have more relevance [20], [21]. In this work a 
fixed RF inductor 4310LC-132KEB, with $1.3 \mu \mathrm{H}$ [22] was employed, and Fig. 8 shows its impedance curve.


Fig. 8. Fixed RF inductor 4310LC-132KEB, impedance in function of frequency curve - red line.

\section{E. Associating cross dipoles and matryoshka geometries}

Cross dipole and matrioska geometries show a typical frequency response as illustrated in Fig. 9(a) and (b), respectively. By associating the two geometries, the frequency response presented in $9(\mathrm{c})$ is obtained. It should be noted that these results are only illustrative, without compromise between the values of the resonant frequencies and the geometry dimensions.

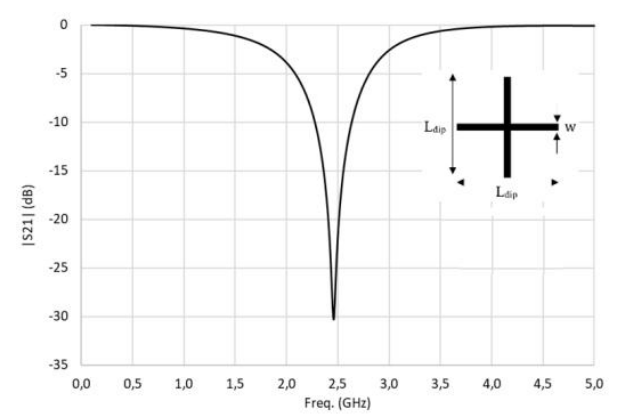

a)

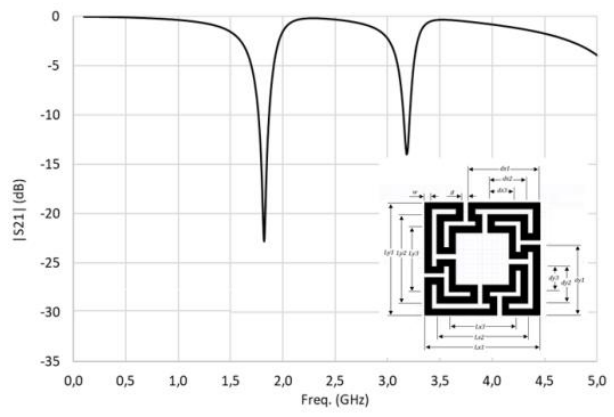

b)

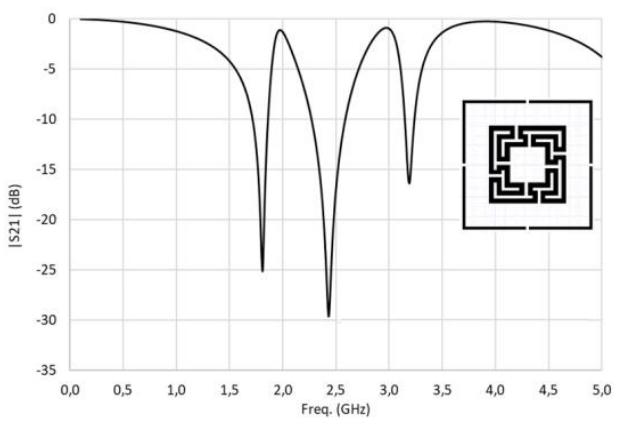

c)

Fig. 9. Typical frequency responses: a) Cross dipoles frequency response, b) Matryoshka frequency response, c) Associating cross dipoles and matryoshka geometries

In a previous work [23], a reconfigurable FSS associating cross dipoles and matryoshka geometries was described, with the cross dipoles'horizontal arms short-circuited and PIN diodes inserted into the vertical arms, as seen in Fig. 10. In this case, for the $X$ polarization the cross dipoles resonance is lost, despite the achieved reconfigurability for the $y$ polarization. This is observed in Fig. 11. 




Fig. 10. FSS unit cell with the cross dipoles' horizontal arms short-circuited and PIN diodes inserted into the vertical arms.
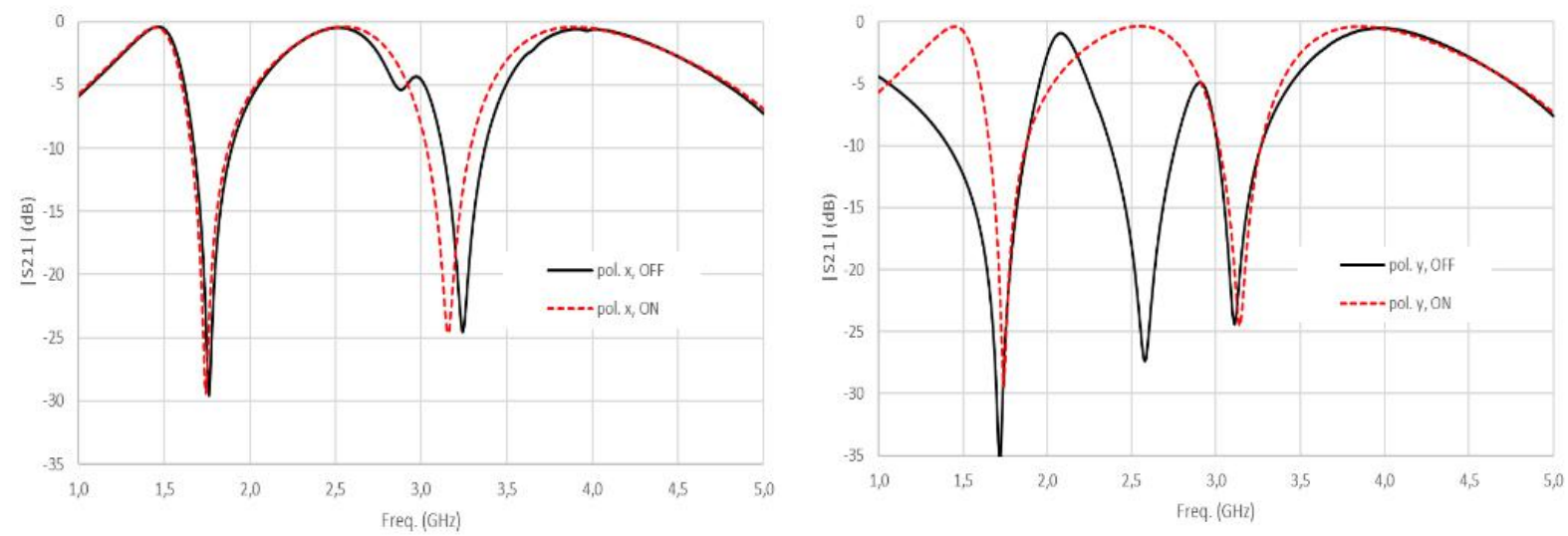

Fig. 11. Typical reconfigurable FSS frequency responses for cross dipoles' horizontal arms short-circuited and PIN diodes inserted into the vertical arms.

After [23], in this paper the short-circuit in cross dipoles horizontal arms is replaced by a RF inductor like is seen in Fig. 12. The main objective is to achieve the reconfigurability for $y$ polarization, but keeping the cross dipoles resonance for $X$ polarization.



Fig. 12. FSS unit cell with RF inductors replacing the short-circuit.

\section{NUMERICAL AND MEASURED RESULTS}

Numerical results were obtained using the commercial software ANSYS HFSS [24]. The measured results were acquired at the GTEMA/IFPB microwave measurements laboratory using an Agilent E5071C two ports network analyzer [25], two double ridge guide horn antennas SAS 571 [26], and a measurement window $(80 \mathrm{~cm} \times 80 \mathrm{~cm} \times 0.3 \mathrm{~cm}$ aluminum sheet, with $20 \mathrm{~cm} \times 20 \mathrm{~cm}$ center opening) as shown in Fig. 13. Each unit cell has $30 \mathrm{~mm} \times 30 \mathrm{~mm}(W x \times W y)$, and the substrate is a low-cost fiber-glass FR-4 with $\varepsilon_{r}=4.4$, loss tangent $\operatorname{tg}(\delta)=0.02$, thickness $h=1.6 \mathrm{~mm}$. For $x$ 
and $y$ polarizations, the wave incidence is considered normal to the FSS. As previously mentioned, the PIN diode BAR 64-03W [19] and the fixed RF inductor 4310LC-132KEB [22] were employed.

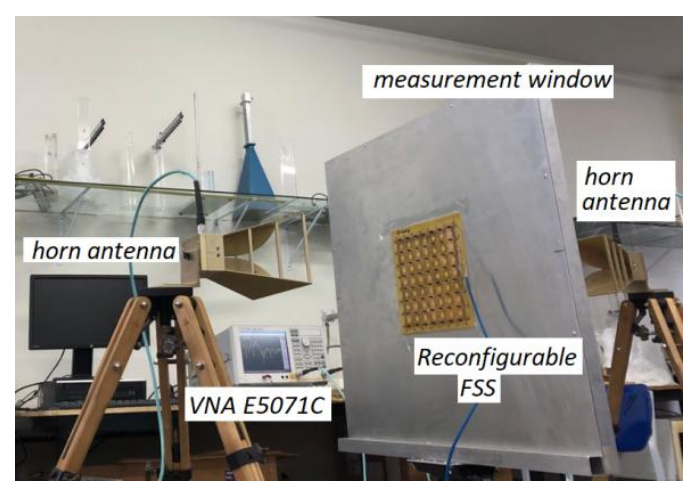

Fig. 13. Measurement setup.

The fabricated reconfigurable FSS has $7 \times 7$ unit cells, corresponding to a whole dimension of $210 \mathrm{~mm} \times 210 \mathrm{~mm}$. The PIN diodes were biased by an arrangement of 8 equal resistors (150 ohms), as shown in Fig. 14. Table I presents a summary of the dimensions of the cross dipoles and matryoshka geometries.

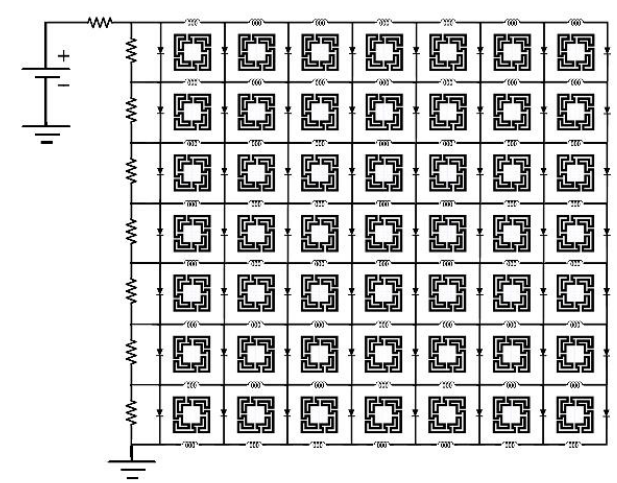

a)

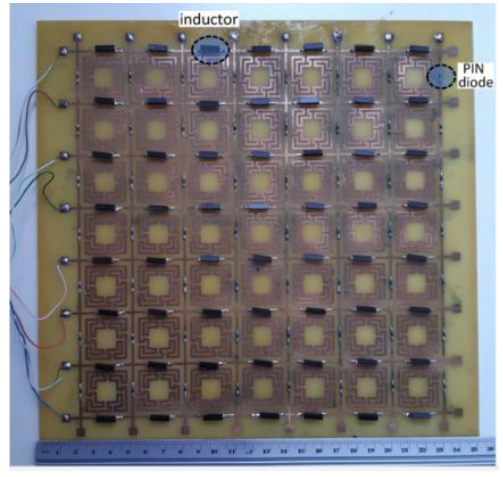

b)

Fig. 14. Bias circuit schematic and fabricated prototype: a) Bias circuit schematic, b) Fabricated prototype.

TABLE I. DIMENSIONS OF THE FSS UNIT CELL

\begin{tabular}{cccccc}
\hline $\boldsymbol{W} \boldsymbol{x}$ & $30.0 \mathrm{~mm}$ & $\boldsymbol{g}$ & $1.0 \mathrm{~mm}$ & $\boldsymbol{d 1}$ & $15.0 \mathrm{~mm}$ \\
$\boldsymbol{W} \boldsymbol{y}$ & $30.0 \mathrm{~mm}$ & $\boldsymbol{L 1}$ & $24.0 \mathrm{~mm}$ & $\boldsymbol{d} 2$ & $8.5 \mathrm{~mm}$ \\
$\boldsymbol{L d i \boldsymbol { p }}$ & $29.0 \mathrm{~mm}$ & $\boldsymbol{L 2}$ & $19.0 \mathrm{~mm}$ & $\boldsymbol{d} 3$ & $6.0 \mathrm{~mm}$ \\
$\boldsymbol{w}$ & $1.5 \mathrm{~mm}$ & $\boldsymbol{L 3}$ & $14.0 \mathrm{~mm}$ & $\boldsymbol{h}$ & $1.6 \mathrm{~mm}$ \\
\hline
\end{tabular}

Initially, the proposed structure was characterized numerically and experimentally without the RF inductors and PIN diodes, and Fig. 15 provides the results [23]. As expected, numerically the frequency responses for the $x$ and $y$ polarizations are the same, with only the answer for the $y$ polarization being presented. We have to keep in mind that when we use Floquet's port we are considering a perfect infinite and periodic structure, and it is not the real measurement scenario. This difference between the real and numerical scenarios becomes more important mainly in the results 
that involve higher order modes. Thus, a good agreement is observed between numerical and experimental results, verifying three resonances. Table II summarizes the values obtained.
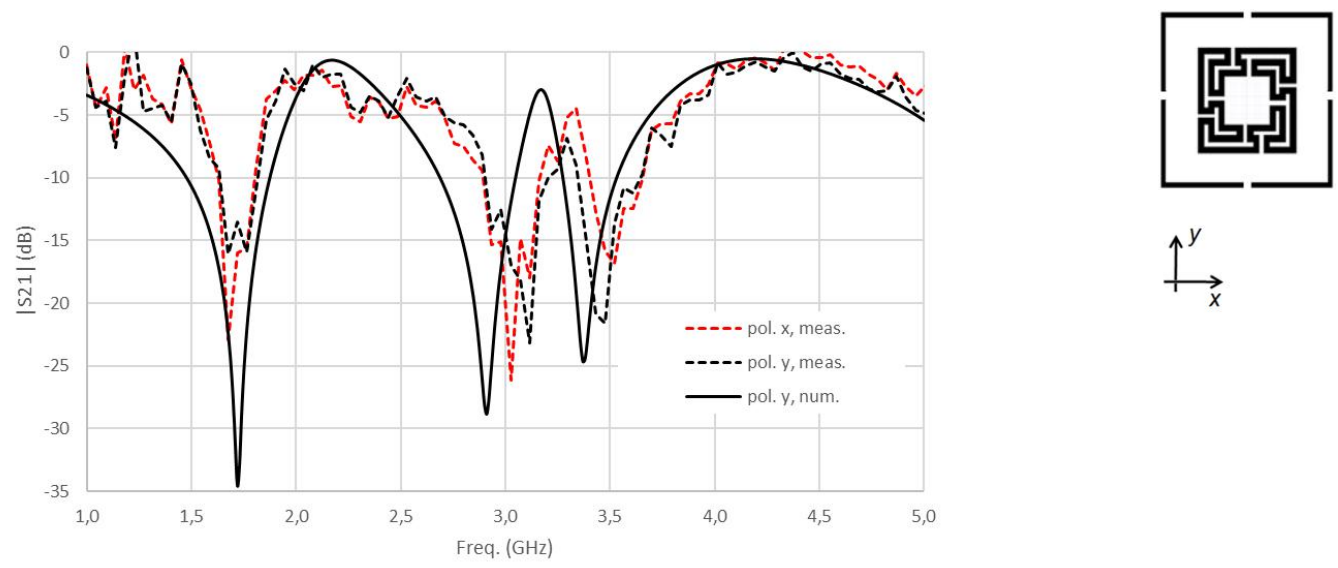

Fig. 15. Reconfigurable FSS frequency response, without PIN diodes and RF inductors.

TABLE II - RESONANT FREQUENCIES - RECONFIGURABLE FSS WITHOUT PIN DIODES AND RF INDUCTORS

\begin{tabular}{clcccc}
\hline \multicolumn{1}{c}{ Geometry } & $\begin{array}{c}\text { Num. } \\
\text { (ANSYS }\end{array}$ & $\begin{array}{c}\text { Calculated } \\
\text { (Eqs. (1) - (6)) }\end{array}$ & $\begin{array}{l}\text { Meas. } \\
\text { pol. } \boldsymbol{x}\end{array}$ & $\begin{array}{c}\text { Meas. } \\
\text { pol. } \boldsymbol{y}\end{array}$ \\
\hline $\boldsymbol{f}_{\boldsymbol{r} \text { : }}$ Matryoshka & $1.72 \mathrm{GHz}$ & $1.94 \mathrm{GHz}$ & $1.68 \mathrm{GHz}$ & $1.68 \mathrm{GHz}$ \\
$\boldsymbol{f}_{\boldsymbol{r} \mathbf{2}}$ & Cross dipoles & $2.91 \mathrm{GHz}$ & $3.16 \mathrm{GHz}$ & $3.03 \mathrm{GHz}$ & $3.12 \mathrm{GHz}$ \\
$\boldsymbol{f}_{\boldsymbol{r} \mathbf{3}}$ & Matryoshka & $3.37 \mathrm{GHz}$ & $3.27 \mathrm{GHz}$ & $3.52 \mathrm{GHz}$ & $3.48 \mathrm{GHz}$ \\
\hline
\end{tabular}

Comparing the results calculated for the matryoshka geometry, equations (3) - (6), with the measured results, there is a difference of $15.4 \%$ and $6.0 \%$, in $f_{r 1}$ and $f_{r 3}$, respectively. However, from these values, the desired frequencies can be numerically adjusted without a greater computational effort. For the cross dipoles, equations (1) and (2), the observed difference is approximately $4.0 \%$, a good result for a posterior numerical optimization.

Figure 16 shows the frequency responses $\left(\mathrm{S}_{21}\right)$ for the reconfigurable FSS with the PIN diodes inserted, but without the RF inductors, corresponding to zero bias state. Herein the main objective is to verify PIN diode effects in the reconfigurable FSS frequency responses. In the numerical simulation, boundary Lumped RLC was employed, considering $R=10 \mathrm{k} \Omega$ [24]. A first point to be highlighted is the good agreement between numerical and measured results for the matryoshka resonant frequencies, as can be seen in Table III. For the cross dipoles, the resonant frequencies are observed for both polarizations. However, in the measured results, for $x$ polarization, there is a coupling between the cross dipoles resonant frequency and the second matryoshka resonant frequency and a difference of $9.1 \%$ is observed. 

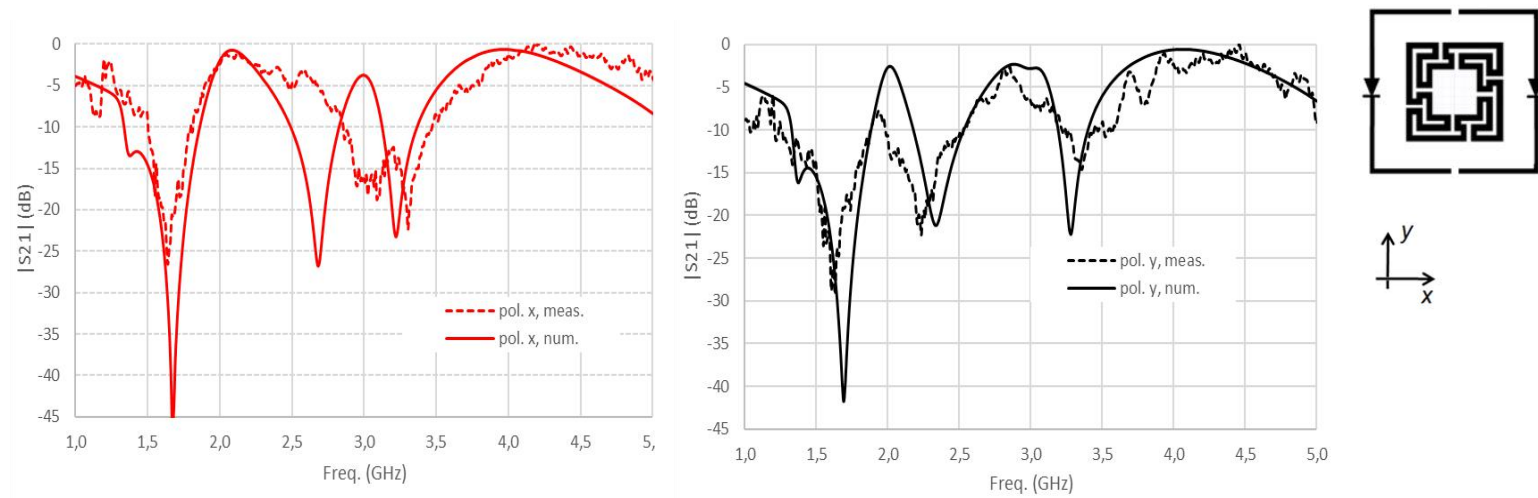

Fig. 16. Reconfigurable FSS frequency response, with PIN diodes, and without RF inductors.

TABLE III - RESONANT FREQUENCIES - RECONFIGURABLE FSS WITH PIN DIODES AND WITHOUT RF INDUCTORS

\begin{tabular}{clllll}
\hline \multicolumn{1}{c}{ Geometry } & $\begin{array}{l}\text { Num. } \\
\text { pol. } \boldsymbol{x}\end{array}$ & $\begin{array}{l}\text { Meas. } \\
\text { pol. } \boldsymbol{x}\end{array}$ & $\begin{array}{c}\text { Num. } \\
\text { pol. } \boldsymbol{y}\end{array}$ & $\begin{array}{c}\text { Meas. } \\
\text { pol. } \boldsymbol{y}\end{array}$ \\
\hline $\boldsymbol{f}_{\boldsymbol{r} \text { : }}$ Matryoshka & $1.67 \mathrm{GHz}$ & $1.64 \mathrm{GHz}$ & $1.69 \mathrm{GHz}$ & $1.63 \mathrm{GHz}$ \\
$\boldsymbol{f}_{\boldsymbol{r} \mathbf{2}}$ & Cross dipoles & $2.68 \mathrm{GHz}$ & $2.95 \mathrm{GHz}$ & $2.34 \mathrm{GHz}$ & $2.21 \mathrm{GHz}$ \\
$\boldsymbol{f}_{\boldsymbol{r} \mathbf{3}}$ & Matryoshka & $3.22 \mathrm{GHz}$ & $3.30 \mathrm{GHz}$ & $3.27 \mathrm{GHz}$ & $3.36 \mathrm{GHz}$ \\
\hline
\end{tabular}

Including the RF inductors, as showed Fig. 12, results for the $x$ polarization are shown in Fig. 17, considering zero $(\mathrm{OFF})$ and forward $(\mathrm{ON})$ bias PIN diode states. In the numerical simulation, boundary Lumped RLC was employed, with $L=1.3 \mu \mathrm{H}$ (RF inductor), $R=10 \mathrm{k} \Omega$ (PIN diode OFF), and $R=100 \Omega$ (PIN diode ON) [24]. The three resonant frequencies are observed for both bias PIN diode states, with a good agreement when compared to numerical results. Table IV summarizes the results.
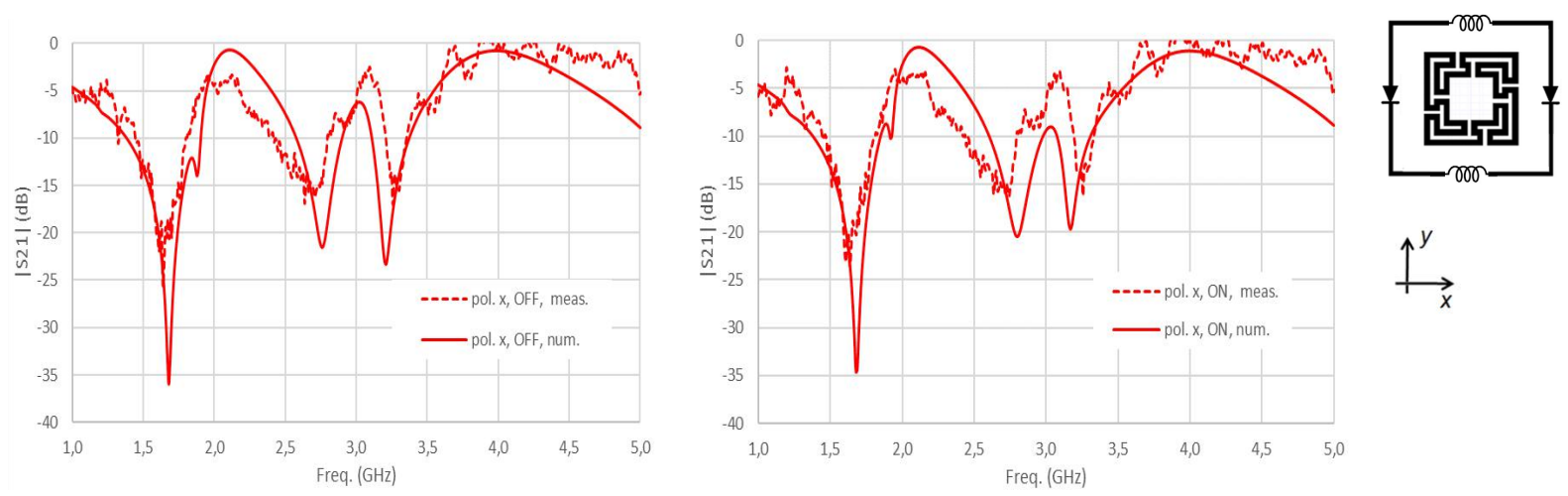

Fig. 17. Reconfigurable FSS frequency response, with PIN diodes and inductors, polarization $x$.

Analogously to the $x$ polarization, in Fig. 18 results are presented considering $y$ polarization. For the OFF state, the three resonant frequencies are verified, and for the ON state, as expected, only the matryoshka resonances are observed, with a good agreement between numerical and measured results. In Table IV the results related to Fig. 17 and Fig. 18 are summarized. 



Fig. 18. Reconfigurable FSS frequency response, with PIN diodes and RF inductors, polarization $y$.

TABLE IV - RESONANT FREQUENCIES - RECONFIGURABLE FSS WITH PIN DIODES AND RF INDUCTORS

\begin{tabular}{|c|c|c|c|c|c|c|c|c|c|}
\hline & Geo & $\begin{array}{l}\text { Num. } \\
\text { pol. } x, \text { off }\end{array}$ & $\begin{array}{c}\text { Meas. } \\
\text { pol. } x, \text { off }\end{array}$ & $\begin{array}{c}\text { Num. } \\
\text { pol. } y, \text { off }\end{array}$ & $\begin{array}{c}\text { Meas. } \\
\text { pol. } y, \text { off }\end{array}$ & $\begin{array}{c}\text { Num. } \\
\text { pol. } x, \text { on }\end{array}$ & $\begin{array}{c}\text { Meas. } \\
\text { pol. } x, \text { on }\end{array}$ & $\begin{array}{c}\text { Num. } \\
\text { pol. } y, \text { on }\end{array}$ & $\begin{array}{c}\text { Meas. } \\
\text { pol. } y \text {, on }\end{array}$ \\
\hline$f_{1}$ & Mat & $\mathrm{Hz}$ & & $z$ & $z$ & $z$ & & $z$ & \\
\hline$f_{r 2}$ & Cros & $2.76 \mathrm{GHz}$ & $2.70 \mathrm{GHz}$ & $2.33 \mathrm{GHz}$ & $2.21 \mathrm{GHz}$ & $2.80 \mathrm{GHz}$ & 2.74 & - & - \\
\hline $\mathrm{r} \mathbf{3}$ & Matryoshka & $21 \mathrm{GHz}$ & $3.26 \mathrm{GHz}$ & $3.29 \mathrm{GHz}$ & $3.29 \mathrm{GHz}$ & $3.18 \mathrm{GHz}$ & $3.25 \mathrm{GHz}$ & $3.28 \mathrm{GHz}$ & $3.29 G H$ \\
\hline
\end{tabular}

In Fig. 19, measured results are compared for both polarizations and bias states. For $x$ polarization, the frequency responses are almost the same for considering ON and OFF states. In other words, the $\mathrm{RF}$ inductor properly isolated cross dipoles horizontal arms. For $y$ polarization, the reconfiguration of the FSS is verified, with a reconfigurable bandwidth of $0.24 \mathrm{GHz}$, from $2.12 \mathrm{GHz}$ to $2.36 \mathrm{GHz}$, for at least $10 d B$ difference between OFF and ON bias states.
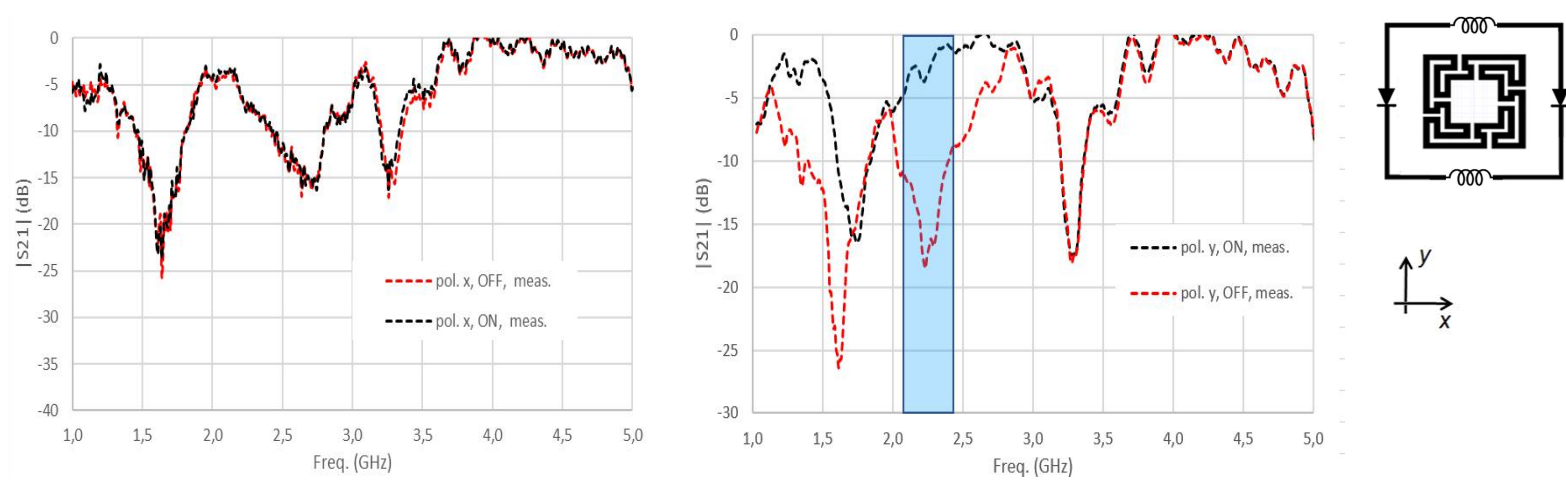

Fig. 19. Reconfigurable FSS frequency response, with PIN diodes and inductors.

\section{CONCLUSIONS}

The development of a reconfigurable FSS associating cross dipoles and matryoshka geometries was described in this paper. Differently from our previous work [23], a RF inductor was inserted in the cross dipoles' horizontal arms. In this way, the cross dipoles resonance for $x$ polarization was maintained. However, the FSS reconfigurability was achieved by the use of PIN diode inserted in the cross dipoles' vertical arms. Therefore, the proposed reconfigurable FSS presents three resonances, two fixed associated to the matryoshka geometry, and one that can be activated or not, associated to the cross dipoles geometry. Numerical and measured results were presented, verifying a good Brazilian Microwave and Optoelectronics Society-SBMO received 28 Jan 2021; for review 28 Jan 2021; accepted 20 Mar 2021 
agreement. For $y$ polarization, the reconfiguration of the FSS was verified, with a reconfigurable bandwidth of $0.24 \mathrm{GHz}$, from $2.12 \mathrm{GHz}$ to $2.36 \mathrm{GHz}$, for at least $10 \mathrm{~dB}$ difference between OFF and $\mathrm{ON}$ bias states. The geometry proposed in this work, as well as the design procedures, can be used for other frequency ranges, with specific applications.

\section{ACKNOWLEDGMENT}

This work was supported in part by the CNPq (Project 407028/2016-1) and CAPES, Brazilian Federal Agencies, and by the IFPB, Federal Institute of Paraíba (PPGEE, PRPIPG 18/2019).

\section{REFERENCES}

[1] B. Marr, "The 7 biggest technology trends that will transform telecoms in 2020", Forbes, Oct. 14, 2019, available in: https://www.forbes.com/sites/bernardmarr/2019/10/14/the-7-biggest-technology-trends-that-will-transform-telecomsin-2020/\#db1345660332.

[2] R. Panwar, J. R. Lee, "Progress in frequency selective surface-based smart electromagnetic structures: A critical review", Aerospace Science and Technology, vol. 66, pp. 216-234, 2017.

[3] Q. Luo, S. Gao, B. S. Izquierdo, X. Yang, X. Ren and J. Wu, "Low-cost smart antenna using active frequency selective surfaces", 2019 International Symposium on Antennas and Propagation (ISAP), Xi'an, China, 2019, pp. 1-3.

[4] Q. Guo, Z. Li, J. Su, J. Song and L. Y. Yang, "Active frequency selective surface with wide reconfigurable passband", IEEE Access, vol. 7, pp. 38348-38355, 2019.

[5] U. Farooq, M. F. Shafique and M. J. Mughal, "Polarization insensitive dual band frequency selective surface for RF shielding through glass windows", IEEE Transactions on Electromagnetic Compatibility, vol. 62, no. 1, pp. 93-100, Feb. 2020.

[6] S. Cho, I. Lee and I. Hong, "Frequency selective film design for building walls for blocking wireless LAN signal", 2018 International Symposium on Antennas and Propagation (ISAP), Busan, Korea (South), 2018, pp. 1-2.

[7] R. S. Anwar, L. Mao and H. Ning, "Frequency Selective Surfaces: A Review", Applied Sciences, vol. 8, no. 9, 1689, Sep. 2018.

[8] B. A. Munk, Frequency Selective Surfaces - Theory and Design, New York: Wiley, 2000.

[9] A. Gomes Neto, J. C. e Silva, I. B. G. Coutinho, M. de O. Alencar, and D. M. de Andrade, "Triple band reject frequency selective surface with application to $2.4 \mathrm{GHz}$ band", JCIS, vol. 35, no. 1, pp. 77-85, Apr. 2020.

[10] A. Gomes Neto, J. C. e Silva, I. B. G. Coutinho, M. de O. Alencar, I. de F. Albuquerque, B. L. G. dos Santos, "Polarization independent triple-band frequency selective surface based on matryoshka geometry", 2019 SBMO/IEEE MTT-S International Microwave and Optoelectronics Conference (IMOC), Aveiro, Portugal, Nov. 2019.

[11] R. Simons, Coplanar Waveguide Circuits, Components, and Systems, USA: Wiley, 2001.

[12] http://www.hp.woodshot.com.

[13] https://www.emtalk.com/mscalc.php

[14] H. de P. A. Ferreira, Uma Proposta de Geometria para FSS Multibandas, in Portuguese, Master thesis, PPGEE, IFPB, João Pessoa, PB, Brazil, 2014.

[15] A. Gomes Neto, A. G. DAssunção, J. C. e. Silva, A. N. d. Silva, H. d. P. A. Ferreira and I. S. S. Lima, "A proposed geometry for multi-resonant frequency selective surfaces", 2014 44th European Microwave Conference, Rome, 2014, pp. 897-900. doi: 10.1109/EuMC.2014.6986580.

[16] A. Gomes Neto, T. R. de Sousa, J. C. E. Silva and D. F. Mamedes, "A polarization independent frequency selective surface based on the matryoshka geometry", 2018 IEEE/MTT-S International Microwave Symposium - IMS, Philadelphia, PA, 2018, pp. 999-1002.

[17] T. R. de Sousa, Desenvolvimento de Superfícies Seletivas em Frequência Baseadas na Geometria Matrioska Independente da Polarização, in Portuguese, Master thesis, PPGEE, IFPB, João Pessoa, PB, Brasil, 2019.

[18] Skyworks Solutions, Inc., Design with PIN Diodes - Application Note, Oct. 2012.

[19] Infineon-BAR64series-DS-v01_02-EN-1107806.pdf. Available in: https://br.mouser.com/datasheet/2/196/InfineonBAR64series-DS-v01 02-EN-1107806.pdf.

[20] L. Green, "RF-inductor modeling for the 21st century", EDN, September 2001, pp. 67-74.

[21] Coilcraft Inc., "Basic of design - Investigating Inductor Requirements for Power and RF/Microwaves Designs", Electronic Design Magazine, March 2014. Available in: https://www.coilcraft.com/getmedia/0216fb39-cc39-4cd9a5b2-1578ce1c204e/Doc1189_Coilcraft_Basics-of-Inductor-Selection.pdf

[22] https://www.coilcraft.com/en-us/products/rf/conical-broadband/0-6-ghz/4310lc/\#

[23] A. Gomes Neto, J. C. e Silva, I. B. G. Coutinho, D. C. G. Fernandes, L. M. de S. Duarte, A. G. Barboza, "Desenvolvimento de superfície seletiva em frequência reconfigurável associando as geometrias dipolos cruzados e matrioska", in Portuguese, $19^{\circ}$ SBMO - Simpósio Brasileiro de Micro-ondas e Optoeletrônica e o $14^{\circ}$ CBMagCongresso Brasileiro de Eletromagnetismo, Niterói, RJ, 2020.

[24] http://www.ansys.com.

[25] https://www.keysight.com/br/pt/product/E5071C/e5071c-ena-vector-network-analyzer.html

[26] https://www.ahsystems.com/catalog/SAS-571.php

Brazilian Microwave and Optoelectronics Society-SBMO

received 28 Jan 2021; for review 28 Jan 2021; accepted 20 Mar 2021 Brazilian Society of Electromagnetism-SBMag 